\title{
Itinerários terapêuticos dos pacientes com câncer de cabeça e pescoço: revisão integrativa da literatura
}

Itineraries of patients with head and neck cancer: integrative literature review

Itinerarios terapéuticos de pacientes con cáncer de cabeza y cuello: revisión integrativa de la literatura therapeutic

Cíntia Hein de Oliveira CUNHA ${ }^{1}$

Rafaela Hartmann KASPER1

Gabriela Moraes MACHADO ${ }^{1}$

Caren Serra BAVARESCO ${ }^{2}$

${ }^{1}$ Acadêmica do Curso de Odontologia - ULBRA Univ. Luterana do Brasil, 92425-020 Canoas - RS, Brasil

${ }^{2}$ Cirurgiã Dentista. Doutora em Ciências Biológicas: Bioquímica - UFRGS. Professora do Curso de Graduação em Odontologia ULBRA Univ. Luterana do Brasil, 92425-020 Canoas - RS, Brasil

\section{Resumo}

Introdução: No Brasil, pacientes com câncer de cabeça e pescoço percorrem diversas instâncias de saúde de forma equivocada. Conseguindo a adesão ao tratamento apropriado, em muitos casos, de forma tardia. Objetivo: Realizar uma revisão integrativa sobre o itinerário terapêutico de pacientes com câncer de cabeça e pescoço na rede de atenção básica. Material e método: Foram realizadas buscas na base de dados MEDLINE, PubMed, LILACS, Scielo e Google Acadêmico, tendo como descritores os termos: "therapeutic itinerary", "head and neck cancer", "Health Unic System". Foram excluídas revisões de literatura e artigos sem relação com o tema. Resultados: Todos artigos selecionados observaram a demora no diagnóstico e tratamento. Na maioria dos casos de carcinoma de cabeça e pescoço que são atendidos dentro da rede de atenção básica à saúde, o diagnostico ocorre tardiamente e/ou erroneamente. Conclusão: Observou-se que a demora do diagnóstico e tratamento está ligada basicamente a dois fatores: à falhas no sistema de saúde e a reação e decisões do paciente frente a sua saúde. Pudemos observar que existem grandes falhas no sistema de saúde, como a comunicação. Essas falhas contribuem para o atraso no diagnóstico gerando certo descaso com a vida dos pacientes. Por outro lado, notamos que outra boa parte do processo de cura e descobrimento da doença está ligado com o interesse e reação dos usuários diante do seu diagnóstico, suas decisões de curas alternativas ou desinteresse de busca por saúde agravam o seu estado.

Descritores: Neoplasias de Cabeça e Pescoço; Acesso aos Serviços de Saúde; Sistema Único de Saúde.

\section{Abstract}

Introduction: In Brazil, patients with head and neck cancer go through different health instances in the wrong way. The patients achieve adherence to appropriate treatment, in many cases, late. Objective: To carry out an integrative review on the therapeutic itinerary of patients with head and neck cancer in the primary care network. Material and method: Searches were carried out in the MEDLINE, PubMed, LILACS, Scielo and Google Scholar databases, using the terms: "therapeutic itinerary", "head and neck cancer", "Health Unic System". Literature reviews and articles unrelated to the topic were excluded. Results: All selected articles observed the delay in diagnosis and treatment. In most cases of head and neck carcinoma that are treated within the primary health care network, the diagnosis occurs late and / or erroneously. Conclusion: It was observed that the delay in diagnosis and treatment is basically linked to two factors: failures in the health system and the reaction and decisions of the patient regarding their health. We were able to observe that there are major flaws in the health system, such as communication. These failures contribute to the delay in diagnosis, generating a certain disregard for the patients' lives. On the other hand, we noticed that another good part of the disease healing and discovery process is linked to the users' interest and reaction to their diagnosis, their decisions about alternative cures or lack of interest in seeking health aggravate their condition. Descriptors: Head and Neck Neoplasms; Health Services Accessibility; Unified Health System.

\section{Resumen}

Introducción: en Brasil, los pacientes con cáncer de cabeza y cuello pasan por diferentes instancias de salud de manera incorrecta. Lograr la adherencia al tratamiento adecuado, en muchos casos, tarde. Objetivo: realizar una revisión integradora del itinerario terapéutico de pacientes con cáncer de cabeza y cuello en la red de atención primaria. Material y método: Se realizaron búsquedas en las bases de datos MEDLINE, PubMed, LILACS, Scielo y Google Scholar, utilizando los términos: "itinerario terapéutico", "cáncer de cabeza y cuello", "Sistema Único de Salud". Se excluyeron las revisiones de literatura y artículos no relacionados con el tema. Resultados: Todos los artículos seleccionados observaron el retraso en el diagnóstico y el tratamiento. En la mayoría de los casos de carcinoma de cabeza y cuello que se tratan dentro de la red de atención primaria de salud, el diagnóstico se produce de manera tardía y / o errónea. Conclusión: se observó que la demora en el diagnóstico y el tratamiento está básicamente relacionada con dos factores: fallas en el sistema de salud y la reacción y las decisiones del paciente con respecto a su salud. Pudimos observar que existen fallas importantes en el sistema de salud, como la comunicación. Estas fallas contribuyen a la demora en el diagnóstico, generando un cierto desprecio por la vida de los pacientes. Por otro lado, notamos que otra buena parte del proceso de curación y descubrimiento de la enfermedad está relacionada con el interés y la reacción de los usuarios a su diagnóstico, sus decisiones sobre curas alternativas o la falta de interés en buscar salud agravan su condición.

Descriptores: Neoplasias de Cabeza y Cuello; Accesibilidad a los Servicios de Salud; Sistema Único de Salud.

INTRODUÇÃO

Carcinoma é o termo usado para definição de um conjunto de alterações que têm em comum o crescimento desordenado de células, podendo ter origem epitelial ou no tecido conjuntivo. Quando essas alterações e crescimentos desordenados se iniciam em tecidos epiteliais, como pele ou mucosas, são denominados carcinomas ${ }^{1}$. Por outro lado, se o ponto de partida são os tecidos conjuntivos, como osso, músculo ou cartilagem, estes são chamados sarcomas. Essas células se dividem e multiplicam atingindo e invadindo tecidos e órgãos (metástases), formando tumores e podem se espalhar para outras regiões do corpo ${ }^{1}$.

O câncer é uma das enfermidades mais complexas que existem no quadro atual de doenças, sendo que o seu diagnóstico e 
tratamento são dificultados por muitos fatores distintos, fazendo com que se torne um grande problema para a população e de difícil resolução pelo próprio sistema de saúde, dada a sua magnitude epidemiológica, social e econômica. Cada vez mais, o governo vem interagindo com a população através de programas sociais de prevenção, orientação e conscientização sobre sinais, sintomas e sobre os aspectos gerais da doença. Em média ao menos um terço dos casos de câncer que ocorrem anualmente no mundo poderiam ser prevenidos ${ }^{1}$.

Câncer de cabeça e pescoço é um termo genérico que representa as neoplasias malignas das vias aerodigestivas superiores como cavidade oral, laringe, faringe e seios paranasais $^{2}$. Os sintomas típicos incluem aparecimento de nódulos, feridas que não cicatrizam, dor de garganta que não melhora, dificuldade de engolir, alteração ou rouquidão na voz. Os fatores de risco mais importantes para esse tipo de câncer são o etilismo e tabagismo ${ }^{3}$. A opção de tratamento primordial é terapia cirúrgica e sua utilização seguida por radioterapia é normalmente a prática optada de tratamento em fases iniciais da doença ${ }^{3}$.

Em estudos do banco de dados do DATASUS, onde foram avaliados os fatores de risco, estadiamento da doença e incidência num período de 2000 a 2008, os resultados dessas neoplasias foram de 11,5 casos para cada 100.000 habitantes, na proporção de 6 acometimento de 5 homens e 1 mulher $^{2}$. São consideradas medidas de prevenção à doença: suspensão do cigarro, limitação do consumo de álcool, evitar a exposição à fumaça do cigarro, a carcinogênicos ambientais, detectar precocemente infecção por HPV, manter a saúde bucal, aderir a bons hábitos alimentares e controlar o stress podem ser medidas de prevenção da doença ${ }^{3}$.

No Brasil, o sistema de saúde é descentralizado, pois almeja promover melhor atendimento à população, uma vez que cada nível de atenção corresponde a um determinado conjunto de serviços assistenciais. Nacionalmente, o paciente é amparado pela Política Nacional de Prevenção e Controle do Câncer, que descreve a necessidade de se garantir o cuidado integral ao usuário na Rede de Atenção à Saúde, estabelecendo que o tratamento do câncer será realizado em estabelecimentos de saúde habilitados, como Unidade de Assistência de Alta Complexidade em Oncologia (Unacon) ou como Centro de Assistência de Alta Complexidade em Oncologia (Cacon) ${ }^{4}$.
A rede de serviços de atenção à saúde abrange a atenção básica, atenção secundária e atenção terciária. A atenção básica destacase como o primeiro contato com a rede de atenção e é a principal entrada para o sistema de saúde, sendo constituída por uma equipe multidisciplinar que atende e supre as necessidades da população, através de um atendimento coordenado e integrado. Seguindo os níveis de complexidade, temos a atenção secundária, onde estão as unidades de atendimentos (UPAs), hospitais gerais e outras unidades de atendimento especializado ou de média complexidade. Há também a atenção terciária para onde são encaminhados os casos mais complexos do sistema ${ }^{4}$.

Cabe às secretarias estaduais e municipais de Saúde organizarem o atendimento dos pacientes na rede assistencial primária, os pontos de atenção secundária nas microrregiões sanitárias e os pontos de atenção terciária nas macrorregiões sanitárias, definindo a rota a ser percorrida pelo paciente dentro do sistema de saúde. Essa trajetória pela qual o paciente transita é chamada de itinerário terapêutico ${ }^{5}$.

Todavia, percebe-se que, muitas vezes, os pacientes aderem ao tratamento adequado tardiamente, pois equivocadamente, percorreram diversas instâncias de saúde que não eram adequadas às suas necessidades ${ }^{6}$. Desta forma, o objetivo do presente estudo foi verificar a literatura publicada acerca dos itinerários terapêuticos dos pacientes com câncer de cabeça e pescoço, a fim de elucidar suas trajetórias dentro das redes de atenção à saúde.

MATERIAL E MÉTODO

Para o desenvolvimento deste estudo foi realizada uma revisão integrativa, visando conhecer o que existe na literatura científica nacional e internacional sobre a temática "itinierário terapêutico de pacientes com câncer de cabeça e pescoço e rede de atenção". As buscas das evidências científicas foram realizadas nas bases de dados, Scielo, Google Acadêmico e Pubmed, durante os meses de maio, julho, agosto e setembro de 2019. Para busca de artigos, foram utilizados os termos atualizados nos Descritores em Ciências da Saúde (DeCS), na língua inglesa, sendo eles "therapeutic itinerary", "head and neck cancer", "Health Unic System". Ainda, com o objetivo de uma projeção maior dos resultados desta investigação, foi feita pesquisa junto ao Google Acadêmico com os termos na língua portuguesa (carcinoma de cabeça e pescoço) para abarcar 
a literatura cinza, abrangendo, além de artigos indexados, teses, dissertações e trabalhos de conclusão de curso e revistas periódicas. Os títulos e resumos de todos os artigos encontrados foram lidos por dois pesquisadores de forma independente. Os critérios de inclusão foram: pesquisas oriundas de dados primários de qualquer delineamento cuja o público alvo seja usuários do sistema único de saúde. Os critérios de exclusão foram: estudos que não sejam específicos para câncer bucal, revisões de literatura, e aqueles onde não foi possível identificar relação com a temática. Ao final da categorização e análise dos estudos, foi realizada a interpretação dos achados, cuja síntese apresenta-se em tabela com o ano da publicação, local da realização da pesquisa, metodologia empregada e resultados encontrados.

\section{RESULTADOS}

A partir da busca nas bases de dados estabelecidas, obtiveram-se 188 estudos. Após leitura de todos os títulos e resumos, respeitando-se os critérios de inclusão e exclusão, excluiu-se 183 estudos, totalizando, então, 5 estudos para leitura na íntegra. Para análise descritiva, foram incluídos apenas 5 estudos, sendo 183 artigos excluídos por não se aplicarem ao tema.

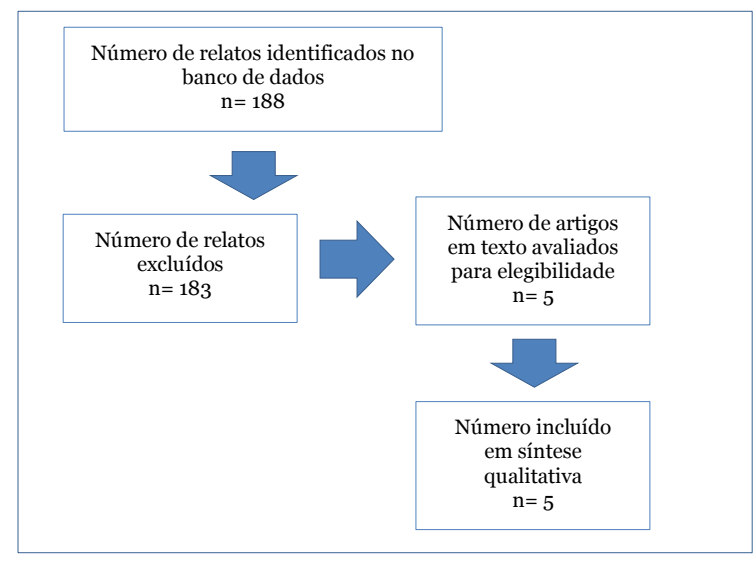

Figura 1: Fluxograma dos resultados.

$\mathrm{Na}$ Tabela 1, pode ser observada, de forma resumida, a evidência científica incluída no estudo, acrescentando as informações relativas aos autores, amostra, metodologia e resultados.

DISCUSSÃO

No presente estudo, identificou-se 5 artigos que buscavam revelar 0 itinerário terapêutico dos pacientes dentro da rede de atenção ao câncer de cabeça e pescoço no âmbito público e privado. Em todos os artigos, foi possível identificar uma fragmentação dos itinerários terapêuticos, os quais são conduzidos de forma heterogênea entre as unidades da federação.

Tabela 1. Categorização e análise dos estudos segundo autor, ano, país, amostra, metodologia e resultados

\begin{tabular}{|c|c|c|}
\hline $\begin{array}{l}\text { AUTOR, } \\
\text { ANO, PAIIS }\end{array}$ & $\begin{array}{c}\text { AMOSTRA E } \\
\text { METODOLOGIA }\end{array}$ & RESULTADOS \\
\hline $\begin{array}{l}\text { Dubow et al. } \\
2011^{6} \\
\text { Brasil }\end{array}$ & $\begin{array}{l}\text { Tipo de Estudo: Pesquisa } \\
\text { qualitativa, descritiva com } \\
\text { abordagem qualitativa. } \\
\text { Amostra: Participaram do } \\
\text { estudo 17 pessoas que foram } \\
\text { diagnosticadas no período } \\
\text { de março abril de 2011. } \\
\text { Objetivo: investigar e } \\
\text { caracterizar o fluxo de } \\
\text { cuidado a esses sujeitos da } \\
\text { perspectiva da linha de } \\
\text { cuidado, nos diferentes } \\
\text { níveis de gestão e atenção. }\end{array}$ & $\begin{array}{l}\text { Os resultados revelaram fragmentação } \\
\text { do cuidado, apontando a necessidade } \\
\text { de reestruturação dos modelos } \\
\text { assistenciais vigentes, por meio da } \\
\text { garantia de um fluxo de cuidado que } \\
\text { opere de modo interligado. } \\
\text { A pesquisa revelou a existência de } \\
\text { deficiências no que diz respeito às } \\
\text { condições operacionais necessárias à } \\
\text { integração dos diversos níveis de } \\
\text { complexidade do sistema, com } \\
\text { consequente desarticulação a e } \\
\text { fragmentação no cuidado a esses } \\
\text { indivíduos. } \\
\text { Também foi possível evidenciar a } \\
\text { influência que os modos de acesso e } \\
\text { modelos de estruturação dos serviços } \\
\text { têm sobre o processo de cuidado, } \\
\text { evidenciando a necessidade de } \\
\text { reorientação por meio da inserção de } \\
\text { novas práticas de gestão e atenção em } \\
\text { saúde direcionadas à integralidade da } \\
\text { atenção nos vários níveis do sistema }\end{array}$ \\
\hline $\begin{array}{l}\text { Berguer et } \\
\text { al. 20147 } \\
\text { Brasil }\end{array}$ & $\begin{array}{l}\text { Tipo de Estudo: Pesquisa } \\
\text { qualitativa, de caráter } \\
\text { descritivo exploratório. } \\
\text { Amostra: } 22 \text { usuários. } \\
\text { Objetivos: Avaliar o } \\
\text { itinerário terapêutico e a } \\
\text { utilização dos serviços de } \\
\text { saúde. }\end{array}$ & $\begin{array}{l}\text { Metade dos entrevistados procurou os } \\
\text { serviços de atenção básica com porta } \\
\text { de entrada. Acesso do serviço terciário } \\
\text { pelas 'portas invisíveis": acesso direto } \\
\text { através deste nível de atenção. } \\
\text { O tempo de espera, para consulta } \\
\text { especializada e diagnóstico variou } \\
\text { bastante, de acordo com o itinerário } \\
\text { terapêutico percorrido }\end{array}$ \\
\hline $\begin{array}{c}\text { Debus et al. } \\
2018^{8} \\
\text { Brasil }\end{array}$ & 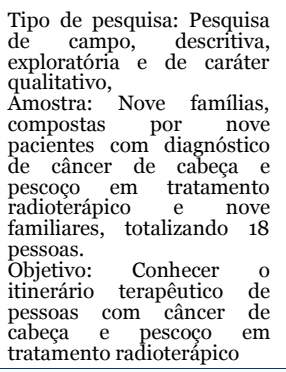 & $\begin{array}{l}\text { O itinerário percorrido pelos pacientes } \\
\text { deu-se nos serviços de saúde do } \\
\text { sistema público e privado, sendo } \\
\text { marcado pela demora para o } \\
\text { diagnóstico e o tratamento. } \\
\text { A busca por alternativas para resolver } \\
\text { o problema de saúde ocorreu também } \\
\text { no meio sociocultural. Torna-se } \\
\text { relevante compreender o contexto em } \\
\text { que a experiência de adoecimento é } \\
\text { vivenciada e o tempo em que as etapas } \\
\text { do processo de diagnóstico e } \\
\text { tratamento foram desenvolvidas, a fim } \\
\text { de oferecer uma melhor assistência. }\end{array}$ \\
\hline $\begin{array}{c}\text { Ayres et al. } \\
2014^{9} \\
\text { Brasil }\end{array}$ & $\begin{array}{l}\text { Tipo do Estudo: Estudo de } \\
\text { natureza qualitativa. } \\
\text { Amostra: } 22 \text { pacientes. } \\
\text { Objetivos: O presente } \\
\text { estudo teve por objetivo } \\
\text { analisar a integralidade do } \\
\text { cuidado a pacientes com } \\
\text { diagnóstico de cancer de } \\
\text { boca e orofaringe sob a } \\
\text { perspectiva do acesso e da } \\
\text { gestão do cuidado na rede } \\
\text { de saúde }\end{array}$ & 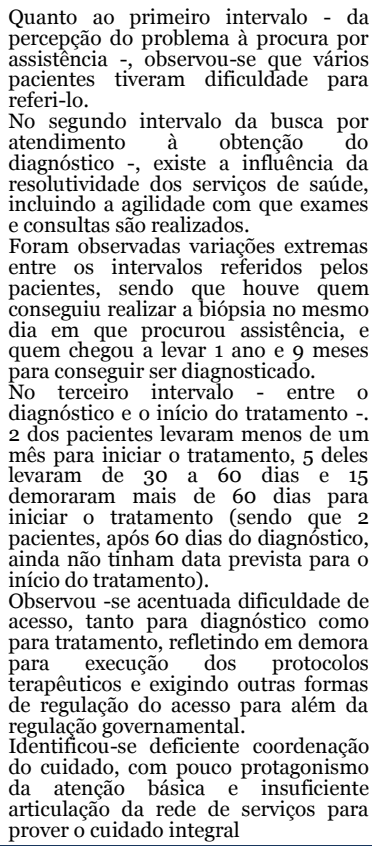 \\
\hline $\begin{array}{c}\text { Souza et al, } \\
2017^{10} \\
\text { Brasil }\end{array}$ & $\begin{array}{l}\text { Tipo de Estudo: Pesquisa de } \\
\text { natureza transversal, } \\
\text { descritiva e exploratória, de } \\
\text { base quantitativa e } \\
\text { qualitativa. } \\
\text { Amostra: Constatou-se que } \\
92 \text { pessoas preenchiam os } \\
\text { critérios de inclusão para } \\
\text { esta pesquisa. O objetivo } \\
\text { deste estudo foi caracterizar } \\
\text { a organização da Rede de } \\
\text { Atenção à Saúde Bucal para } \\
\text { diagnóstico e tratamento de } \\
\text { câncer de boca (RASB/CB) } \\
\text { no Estado de Santa Catarina } \\
\text { (SC) }\end{array}$ & $\begin{array}{l}\text { Constatou-se que são disponibilizados } \\
\text { serviços para dar resposta às pessoas } \\
\text { acometidas por câncer de boca em } \\
\text { todos os níveis de atenção, no âmbito } \\
\text { do diagnóstico e tratamento. No } \\
\text { entanto, há diferenças em relação à } \\
\text { integração entre estes, sendo que, de } \\
\text { forma geral, os municípios de grande } \\
\text { porte apresentam-se em estágio mais } \\
\text { avançado neste sentido, em } \\
\text { comparação aos de pequeno porte. } \\
\text { Observou-se que há uma tendência de } \\
\text { estruturação da rede, de forma a } \\
\text { proporcionar atenção contínua, } \\
\text { equitativa e integral aos pacientes e de } \\
\text { organizacãao, segundo os princípios da } \\
\text { regionalização em saúde }\end{array}$ \\
\hline
\end{tabular}


Em relação ao perfil dos sujeitos de pesquisa, foi possível identificar estes apresentavam idades que variaram entre $18 \mathrm{e}$ 84 anos. Embora os estudos incluídos tenham relatado a presença de pacientes de ambos os sexos, observa-se uma predominância dos pacientes do sexo masculino conforme apontado pelas estimativas do Instituto Nacional de Câncer (Inca) ${ }^{1}$. Em geral, os tumores de cabeça e pescoço são mais frequentes em homens na faixa dos 60 anos de idade e representam o segundo tipo da doença com maior incidência na população masculina e o quinto mais comum entre as mulheres. Acreditase que esse evento esteja relacionado a ao tabagismo, ao consumo de bebidas alcoólicas e a infecções pelo papiloma vírus humano $(\mathrm{HPV})^{11}$.

Em relação ao itinerário terapêutico, a totalidade dos estudos relata demora em relação tempo decorrido para o diagnóstico e para o início do tratamento, o que pode levar a piora do caso, com a progressão da doença e estabelecimento de um prognóstico sombrio ${ }^{12}$. Estes resultados corroboram com estudo de Cecílio et al. $^{13}$, que afirmam que usuários consideram a atenção básica como o lugar de coisas simples, passagem obrigatória para a obtenção de encaminhamentos, pedidos de exames, medicamentos, ou até documentos para se conseguir os benefícios sociais. Traz também a percepção de que o médico generalista teria pouca resolutividade, como um encaminhador para 0 especialista. Esta percepção está arraigada na concepção errônea obtida do modelo taylorista de saúde importado do modelo de atenção presente Estados Unidos, onde só é valorizada a tecnologia de alto custo e ultra especializada ${ }^{5}$.

Quando o paciente dá entrada ao SUS, ele deve, primeiramente, acessar a atenção primária (APS), também conhecida como atenção básica. A APS é orientada pelos seus atributos essenciais: acesso de primeiro contato, longitudinalidade, integralidade e coordenação do cuidado. Cabe a APS a identificação precoce dos casos de carcinoma de cabeça e pescoço e encaminhamento aos demais níveis de atenção. É a atenção que organiza e administra o uso dos recursos, desde os básicos aos especializados e é nesta etapa que os recursos são regidos e direcionados para a promoção, manutenção e melhora da saúde ${ }^{13}$. Neste contexto, De Carvalho et al. ${ }^{14}$ demonstraram que no país, a estrutura da APS e os processos de trabalho ajudaram a reduzir a taxa de mortalidade do câncer de boca, mas não a taxa de incidência da doença, sendo recomendado expandir os investimentos na APS para evitar mortes relacionadas ao câncer de boca.

O acolhimento é parte fundamental da APS. Ele emergiu das primeiras discussões sobre a reorientação da atenção à saúde, sendo elemento fundamental para uma reviravolta na organização da saúde, da assistência em diversos serviços, acarretando uma modificação do modelo assistencial. A partir do HumanizaSUS, o usuário/paciente é visto em sua integralidade, sendo direcionado para o atendimento de sua queixa principal, mas também para outras necessidades não expressas e, se possível, abordadas por uma equipe multiprofissional ${ }^{15}$.

Nos casos de carcinoma de câncer de cabeça e pescoço, o acolhimento configura-se como uma estratégia de identificação de casos suspeitos e direcionamento para a equipe de saúde. Neste contexto, urge que toda a equipe de saúde esteja apta a identificar lesões prémalignas e malignas na cavidade bucal em conjunto com a equipe de saúde bucal. Seguindo o fluxo da rede de saúde a atenção secundária, ela é formada pelos serviços especializados em nível ambulatorial e hospitalar, com um nível intermediário entre a atenção primária e a terciária, é vista como fonte de procedimentos de média complexidade. Esse nível de atendimento compreende serviços médicos e de saúde especializados, de apoio diagnóstico e terapêutico, bem como atendimento de urgência e emergência ${ }^{16}$.

O papel da saúde secundária corresponde à oferta de tratamento tanto em especialidades, quanto à elaboração de planos de tratamento aos usuários referenciados, por dentistas e médicos especialistas, onde a realização do tratamento é feita na atenção primária após a contra referência do caso, ou seja, funcionando também como um serviço de consultoria $^{17}$.

Os Centros de Especialidades Odontológicas (CEOs) são serviços de atenção secundária, devendo ser reproduzidos em unidades de referência para a Atenção Básica. Devem ser integrados ao processo de planejamento regional e local, que devem suprir minimamente as necessidades nas especialidades de periodontia, endodontia, pacientes com necessidades especiais, diagnóstico bucal e cirurgia oral menor. Em relação ao atraso dentro do itinerário terapêutico dos pacientes, as possibilidades ofertadas pela rede de atenção não são uniformes, estando relacionadas a 
disponibilidade de centros de especialidade para encaminhamento, o que não ocorre, principalmente, em municípios de pequeno porte. O conhecimento do desfecho clínico, dado pelo especialista, somado a organização da atenção dada na atenção primária, faz-se fundamental para 0 estabelecimento da coordenação do cuidado. Como pode ser observado nos artigos incluídos no presente estudo, foram observadas variações extremas entre os intervalos referidos pelos pacientes, sendo que houve quem conseguiu realizar a biópsia no mesmo dia em que procurou assistência, e quem chegou a levar 1 ano e 9 meses para conseguir ser diagnosticado. Estes descompassos nos tratamentos podem levar a criação das portas invisíveis como descrito no estudo de Berguer e Willer ${ }^{7}$, em que os pacientes buscam auxílio diretamente no nível terciário?.

O nível terciário é o nível de maior complexidade e abrange os grandes hospitais, nos quais são realizados procedimentos e exames que demandam de equipamentos especializados e avançados ${ }^{4}$.

Muito embora o acesso aos serviços odontológicos tenha melhorado, o padrão de desigualdade no uso de serviços odontológicos entre as unidades da federação e entre os indivíduos pareceu se manter conforme pode ser observado nos artigos descritos no presente estudo, uma vez que o tempo de espera para a consulta especializada, diagnóstico e tratamento variou significativamente, de acordo com o itinerário terapêutico percorrido dentro da localidade de residência do paciente. Outro ponto destacado nos artigos incluídos refere-se à diferença entre a agilidade do serviço público e privado, sendo o serviço privado considerado mais eficaz no direcionamento dos pacientes ${ }^{18}$.

Além disso, Debus et al. $^{8}$ relataram a utilização de recursos naturais e caseiros. $O$ itinerário terapêutico inicia quando acontece o reconhecimento de que o paciente está doente. A longa espera por atendimento, também neste primeiro estágio de percepção da doença, faz com que alguns pacientes busquem, inicialmente, fazer uso de produtos naturais, retardando a procura do serviço de saúde. Desta forma, é importante destacar que, não somente os serviços de saúde devem estar atentos aos primeiros sinais clínicos dos carcinomas de cabeça e pescoço, como também os pacientes e familiares, para que a busca pelo cuidado se inicie precocemente.

CONSIDERAÇÕES FINAIS

Apesar da constante busca por soluções em relação ao estabelecimento das redes de atenção e da qualificação do cuidado na rede pública, os itinerários terapêuticos dos usuários com câncer de cabeça e pescoço ainda são extremamente fragmentados e heterogêneos. Urge que estratégias abrangentes em nível da gestão, dos profissionais e da comunidade sejam realizadas a fim de garantir tratamentos adequados para a população.

\section{REFERÊNCIAS}

1. Brasil. Ministério da Saúde. INCA - Instituto Nacional do Câncer. O que é Câncer? Disponível em: https://www.inca.gov.br/o-quee-cancer. Acesso em: 29 de março 2019

2. Casati MFM, Vasconcelos JA, Vergnhanini GS, Contreiro PF, Graça T, Kanda JL, et al. Epidemiologia do câncer de cabeça e pescoço no Brasil: estudo transversal de base populacional. Rev Bras Cir Cabeça Pescoço. 2012;41(4):186-91.

3. Galbiatti ALS, Padovani-Junior JA, Maníglia JV, Rodrigues CDS, Pavarino ÉC, Goloni-Bertollo EM. Head and neck cancer: causes, prevention and treatment. Braz $\mathrm{J}$ Otorhinolaryngol. 2013;79(2):239-47.

4. Brasil. Ministério da Saúde. Política Nacional de Atenção Básica Departamento de Atenção Básica. Brasília - DF: Ministério da Saúde. Secretaria de Atenção à Saúde, 2012.

5. Mendes EV. As Redes de Atenção à Saúde. Organização Pan-Americana de Saúde; 2011.MENDES, Eugênio Vilaça. As redes de atenção à saúde. Ciênc saúde coletiva. 2010; 15(5):2297-305.

6. Dubow C, Olivo VMF. Análise de fluxo de atenção no Sistema Único de Saúde: elementos para pensar a estruturação de linhas de cuidado em usuários acometidos por agravos neoplásicos de cabeça e pescoço [monografia]. Santa Maria: Centro de Ciências de Saúde, Universidade Federal de Santa Maria; 2011.

7. Berger IC, Willer TH. Itinerário terapêutico de usuários com neoplasia de cabeça e pescoço na rede municipal de atenção em saúde de Santa Maria - RS [monografia]. Santa Maria: Programa de Pós-graduação em Residência Multiprofissional Integrada em Sistema Público de Saúde, Universidade Federal de Santa Maria; 2014.

8. Debus PD, Perlini G, Oliveira NM, da Costa BV, Dalmolin A, Somavilla IM et al. "Entre uma consulta e outra": itinerário terapêutico de pessoas com câncer de cabeça e pescoço. Rev pesqui cuid fundam. 2018;10(4):1032-40.

9. Ayres EC, Andrade MGG. Itinerários terapêuticos de pacientes com câncer de boca e orofaringe: acesso e integralidade do cuidado na Rede de Atenção à Saúde da região de Campinas [dissertação]. Campinas: Faculdade de Ciências Médicas, Universidade Estadual de Campinas; 2014. 
10. Souza ML, Ana LSFM. Rede de atenção à saúde bucal para diagnóstico e tratamento de câncer de boca no estado de Santa Catarina [tese]. Florianópolis: Universidade Federal de Santa Catarina; 2017.

11. Instituto Nacional do Câncer. Julho Verde: INCA debate linha de cuidado e diagnóstico precoce de tumores de cabeça e pescoço. Instituto Nacional do Câncer; 2019. Disponível em: $\quad$ https://www.inca.gov.br/noticias/julhoverde-inca-debate-linha-de-cuidado-ediagnostico-precoce-de-tumores-de-cabeca-e. Acesso em: 10 de outubro de 2019.

12. Felippu D, Wady A, Cesar Freire E, de Arruda Silva R, Guimarães AV, Dedivitis RA. Impacto da demora no diagnóstico e tratamento no câncer de cabeça e pescoço. Braz J Otorhinolaryngol. 2016;82(2):140-43.

13. Cecilio LCO, Andreazza R, Carapinheiro G, Araújo EC, Oliveira LA, Andrade de MGG et al. A Atenção Básica à Saúde e a construção das redes temáticas de saúde: qual pode ser o seu papel? Ciênc Saúde Coletiva. 2012;17(11):2893-902.

14. De Carvalho FFB, Cohen SC, Akerman M. Refletindo sobre o instituído na Promoção da Saúde para problematizar 'dogmas'. Saúde em Debate. 2017;41(3):265-76.

15. Simões ALA, Rodrigues FR, Tavares DMS, Rodrigues LR. Humanização na saúde: enfoque na atenção primária. Texto Contexto Enferm. 2007;16(3):439-44.

16. Erdmann AL, de Andrade SR, de Mello ALSF, Drago LC. A atenção secundária em saúde: melhores práticas na rede de serviços. Rev Latino-Am Enfermagem. 2013;21(Spe):131-39.

17. Figueiredo N, Goes PSAA. Construção da atenção secundária em saúde bucal: um estudo sobre os Centros de Especialidades Odontológicas em Pernambuco, Brasil. Cad Saúde Pública. 2009;25(2):259-67.

18. de Souza RP, Cordeiro FB, Gonzalez FM, Yamashiro I, Junior AJOP, Tornin OS, et al. Carcinoma de seio maxilar: análise de dez casos. Radiol Bras. 2006;39(6):397-400.

19. Câncer no Brasil: presente e futuro. Rev Assoc Med Bras. 20004;50(1):1.

20. Starfield B. Atenção primária: equilíbrio entre necessidades de saúde, serviços e tecnologia. UNESCO Brasil, Ministério da Saúde; 2004.

21. Oncoguia I. Tratamento do Câncer de Cavidade Nasal e Seios Paranasais por Estágio. Instituto Oncoguia; 2017. Disponível em:

http://www.oncoguia.org.br/conteudo/tratament o-do-cancer-de-cavidadenasal-e-seios-

paranasais-por-estagio/8444/506/. Acesso em: 20 de outubro 2019

22. Brehmer LCF, Verdi M. Acolhimento na Atenção Básica: reflexões éticas sobre a
Atenção à Saúde dos usuários. Ciên saúde coletiva. 2010;159(3):3569-578.

23. Coutinho LRP, Barbieri AR, Santos MLM. Acolhimento na Atenção Primária à Saúde: revisão integrativa. Saúde em debate. 2015;39(105):514-24.

24. Hennington ÉA. Acolhimento como prática interdisciplinar num programa de extensão universitária. Cad Saúde Pública. 2005;21(1): 256-65.

25. Carvalho CA, Marsicano JA, Carvalho FS, Sales-Peres A, Bastos JRM, Sales-Peres SH. Acolhimento aos usuários: uma revisão sistemática do atendimento no Sistema Único de Saúde. Arq Ciênc Saúde. 2008;15(2):93-5.

26. Cardoso LS, Cezar-Vaz MR, Costa VZ, Soares JFdS. Acolhimento no trabalho em saúde da família: um estudo qualitativo. CuidArte Enferm. 2009;3(2):149- 155.

27. Brasil. Ministério da Saúde. Acolhimento nas práticas de produção de saúde. Brasília - DF: Ministério da Saúde. Secretaria de Atenção à Saúde, 2010.

28. Goes PSA, Figueiredo N, Neves JC, Silveira FMM, Costa JFR, Pucca GAJ, et al. Avaliação da atenção secundária em saúde bucal: uma investigação nos centros de especialidades do Brasil. Cad Saúde Pública. 2012;28(Spe):81-9.

29. Ministério da Saúde. Política Nacional de Atenção Hospitalar. MINISTÉRIO DA SAÚDE; $2017 . \quad$ Disponível em: http://www.saude.gov.br/atencaoespecializada-e-hospitalar/assistenci ahospitalar/politica-nacional-de-atencaohospitalar-pnhosp. Acesso em: 11 de setembro de 2019

30. Brasil. Conselho Nacional de Secretários de Saúde - CONASS. Assistência de média e alta complexidade no SUS. vol 9. Brasília. 2007.

31. Brasil. Ministério da Saúde. Manual de Implantação e Implementação NIR - Núcleo interno de regulação para Hospitais Gerais Especializados. Brasília - DF: Ministério da Saúde. Secretaria de Atenção à Saúde, 2017.

32. Brasil. Ministério da Saúde. Sistema Único de Saúde (SUS): estrutura, princípios e como funciona. Brasília - DF: Ministério da Saúde. Secretaria de Atenção à Saúde, 2013. Disponível em: http://www.saude.gov.br/sistema-unico-desaude. Acesso em: 23 de outubro. 


\section{CONFLITO DE INTERESSES}

Os autores declaram não haver conflitos de interesse

\section{AUTOR PARA CORRESPONDÊNCIA}

Caren Serra Bavaresco.

Rua Mariz e Barros 219, Bairro Petrópolis, 90690-390 Porto Alegre, RS, Brasil

Telefone: (51) 991686851.

E-mail: c_bavaresco@yahoo.com.br 